\title{
Regularized and Renormalized Bethe-Salpeter Equations: Some Aspects of Irreducibility and Asymptotic Completeness in Renormalizable Theories
}

\author{
D. Iagolnitzer
}

Service de Physique Théorique, CEN-Saclay, F-91191 Gif-sur-Yvette Cedex, France

\begin{abstract}
Results on the links between 2-particle irreducibility and asymptotic completeness are presented in the framework of a renormalized Bethe-Salpeter formalism, introduced recently by $\mathrm{J}$. Bros from an axiomatic viewpoint, for the most simple class of renormalizable theories. These results, which involve the renormalized 2-particle irreducible kernel $G$ (i.e. from the perturbative viewpoint the sum of renormalized Feynman amplitudes of 2-particle irreducible graphs in the channel considered), complement the general quasiequivalence previously established by Bros for regularized (non-renormalized) Bethe-Salpeter kernels. On the one hand, a formal derivation of (2-particle) asymptotic completeness from the irreducibility of $G$ is given. On the other hand, the links between regularized and renormalized kernels are investigated. This analysis provides in particular a converse derivation (up to some assumptions) of the 2-particle irreducibility of $G$ from asymptotic completeness. As a byproduct, it also provides a more explicit justification of previous heuristic derivations by K. Symanzik of integral equations between $F$ and various differences of values of $G$, and a simple alternative derivation of the recently proposed "renormalized" Bethe-Salpeter equation.
\end{abstract}

\section{Introduction}

The usual Bethe-Salpeter equation reads:

$$
F=G+F \circ G \text {, }
$$

where $F$ is the $2 \rightarrow 2$ Green function, $G$ is the 2-particle irreducible ( 2 p.i.) BetheSalpeter kernel in the $2 \rightarrow 2$ channel considered, i.e. is from the perturbative viewpoint the sum of all Feynman amplitudes of 2-particle irreducible graphs, and $F \circ G$ denotes (in momentum space) the Feynman-type convolution integral

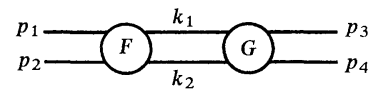

with Feynman propagators or two-point functions attached to each internal line; $p_{1}, p_{2}$ and $p_{3}, p_{4}$ denote the initial and final energy-momenta respectively and 
integration is made over the internal energy-momenta $k_{1}, k_{2}$. As it stands, Eq. (1) is expected to hold in a theory with no renormalization (and correspondingly no convergence problem in the integral $F \circ G$ ). The interest of considering equations such as (1) in more general cases and from an axiomatic, non-perturbative viewpoint has been emphasized in [1], with further analysis and results in [2] and references therein. This program has on the other hand been developed in the complex momentum space formulation in the following two approaches.

First, "regularized" kernels $\hat{G}_{\varrho}$ have been introduced $[3,4]$ in the axiomatic framework and have allowed in particular study of the analytic structure of Green functions in the low energy region in the $2 \rightarrow 2$ case [3] and in the $3 \rightarrow 3$ case [4], with some further steps $[4,5]$ towards the more general multiparticle case (and more general energy regions). A theory with only one (stable) particle, of mass $\mu>0$, is considered for simplicity. In the $2 \rightarrow 2$ case, the only one to be considered in the present work, kernels $\hat{G}_{\varrho}$ are linked to the Green function $F$ via a regularized version of (1):

$$
F=\hat{G}_{\varrho}+F \circ_{\varrho} \hat{G}_{\varrho},
$$

where ${ }_{\varrho}$ is defined as before but with further analytic cut-off factors $\chi_{\varrho}\left(k_{1}\right), \chi_{\varrho}\left(k_{2}\right)$ attached to each internal line, equal to one on-mass-shell and with sufficient decrease at infinity in euclidean directions in order to ensure convergence; e.g. $\chi_{\varrho}\left(k_{i}\right)=e^{-\varrho\left(k_{\imath}^{2}-\mu^{2}\right)^{2}}, \varrho>0$. More usual regularization factors may also be considered, the kernel $\hat{G}_{\varrho}$ depending on the choice of this factor. In the axiomatic framework, $\hat{G}_{\varrho}$ is in fact defined in terms of $F$ through Eq. (2). The kernels $\hat{G}_{\varrho}$ can no longer be expected to have a simple interpretation from the perturbative viewpoint, even formally at $\varrho=0$ if the theory requires renormalization, and are not expected to be well defined in general in this case in the $\varrho \rightarrow 0$ limit. However, a quasi-equivalence, modulo poles arising from zeroes in denominators of solutions of Fredholm-type equations, was proved in [3] between (i) the unitarity or asymptotic completeness equation

$$
F_{+}-F_{-}=F_{+} * F_{-}
$$

in the low energy region $\left(s<(3 \mu)^{2}, s=k^{2}=k_{0}^{2}-\mathbf{k}^{2}, k=p_{1}+p_{2}=p_{3}+p_{4}\right)$, where $*$ now denotes convolution over on-mass-shell values of the internal energymomenta $k_{1}, k_{2}$, and (ii) the axiomatic 2-particle irreducibility of the kernel $\hat{G}_{\varrho}$ (in the $2 \rightarrow 2$ channel considered) in the analytic sense, i.e. analyticity up to the 3-particle threshold. (Thus, there is in particular no singularity at the 2-particle threshold, in contrast to $F$. For the more precise definition, see [3]. This property is known on the other hand to be satisfied by possibly renormalized Feynman amplitudes of 2-particle irreducible graphs.)

On the other hand, a "renormalized" Bethe-Salpeter equation is introduced in [6], so far for the simplest class (beyond the superrenormalizable case) of (scalar) theories in dimension 4 which are just renormalizable and for which the only renormalization parts are the (4-point) vertex parts (and propagators). The 2 p.i. kernel $G$ is from the perturbative viewpoint the sum of all renormalized Feynman amplitudes of 2 p.i. graphs, and the following formal expansion is derived in [6], for the class of theories considered, by a resummation of the perturbative series, 
and is assumed in an axiomatic-type approach:

$$
F=\sum_{n \geqq 0}(\underbrace{G \circ G \ldots \circ G}_{n+1 \text { factors } G})_{r}
$$

Each term $(G \circ G \ldots \circ G)_{r}$ in $(4)$ is a renormalized multiple convolution product in which renormalization does not apply to the internal structure of the kernels $G$ : see Sect. 2. From an axiomatic viewpoint, each term $(G \circ \ldots \circ G)_{r}$ is well defined [6], independently of the perturbative background, modulo suitable assumptions on $G$ which entail in particular that, as in [2], differences of values of $G$ at various points have better decay properties at infinity, in euclidean directions, than $G$ itself. Finally, the renormalized Bethe-Salpeter integral equation is then extracted in [6] from perturbation theory and checked to each order in $G$. For our purposes, we write it here in the following form which (in contrast to [6]) involves an $\varepsilon \rightarrow 0$ limit:

$$
F=G+\lim _{\varepsilon \rightarrow 0}\left\{F \circ_{\varepsilon} G-\Lambda_{\varepsilon}\left[F \circ_{\varepsilon} G\right]\right\},
$$

where

$$
\Lambda_{\varepsilon}=\left(1+\left(F \circ_{\varepsilon} 1\right)\right) \times\left(1+\left[F \circ_{\varepsilon} 1\right]\right)^{-1}
$$

and the notation [ ] means the value of the function when all external energymomenta are fixed e.g. at zero, in accordance with the renormalization prescription used in defining $(G \circ \ldots \circ G)_{r}$. In these equations, $F$ and $G$ are e.g. functions of the variables $k=p_{1}+p_{2}=p_{3}+p_{4}, z=\frac{p_{1}-p_{2}}{2}, z^{\prime}=\frac{p_{3}-p_{4}}{2}$ and $\Lambda_{\varepsilon}$ is a function of $k$ and $z$, which is expected to be defined in the $\varepsilon \rightarrow 0$ limit and admits in fact, at $\varepsilon=0$, the formal expansion:

$$
\Lambda=1+\sum_{n \geqq 1}(\underbrace{G \circ \ldots \circ G}_{n \text { factors } G} \circ 1)_{r},
$$

where $(G \circ \ldots \circ G \circ 1)_{r}$ is defined in the same way as $(G \circ \ldots \circ G)_{r}$ with the last factor $G$ replaced by the (analytic) function 1 . (For $\varepsilon>0, \Lambda_{\varepsilon}$ will admit the same expansion with $\circ$ and $G$ replaced by ${ }_{\varepsilon}$ and $G_{\varepsilon}$, where $G_{\varepsilon}$ is introduced in Sect. 3.) The terms $F \circ_{\varepsilon} G$ and $\left[F \circ_{\varepsilon} G\right]$ are not expected to be individually defined in the $\varepsilon \rightarrow 0$ limit. The renormalized equation has correspondingly been written in [6] in a form in which individual terms are expected to be defined at $\varepsilon=0$, in view in particular of previous assumptions on $G$. Other integral equations between $F$ and differences of values of $G$ at various points have been given in [2]. For applications of these various integral equations and in particular their relation with Wilson short-distance expansion (in space-time) see [2,6].

The aim of this note is to present some results on the extension to renormalized kernels of the analysis of the links between (2-particle) irreducibility and asymptotic completeness, from an axiomatic-type viewpoint. We also wish, in this connection, to analyze the links between regularized and renormalized kernels and, as a byproduct, to give a simple alternative (algebraic) derivation of the renormalized equation, as also a more explicit and satisfactory justification of the heuristic procedure used in [2] to derive the integral equations mentioned above. 
In Sect. 2.1 it is first explained, in an alternative approach to that of [3], how the irreducibility of the regularized kernel $\hat{G}_{\varrho}$ yields the unitarity Eq. (3), in the sense of formal expansions of $F$ in $\hat{G}_{\varrho}$. (A general approach of this type is proposed in [5] in the multiparticle case.) The analysis is then extended to the renormalized case in Sect. 2.2: namely, starting from (4), it is shown how the irreducibility of $G$ yields again Eq. (3), in the sense of formal expansions in $G$. (Complementary results based on integral equations will be found in [6].) Our analysis can be considered as an extension (in the most simple situation) to the case of irreducible kernels of previous results of $[7,8]$ on unitary in perturbation theory, as will be indicated at the end.

In Sect. 3, links between various regularized kernels (Sect. 3.1) and between regularized and renormalized kernels (Sect. 3.2) are established, both from perturbative and non-perturbative viewpoints. These links explain in a direct way why the irreducibility (in the axiomatic sense) of one of these kernels should yield the irreducibility of the other ones. In particular, the following result (reminiscent of well known facts on Feynman amplitudes but not a priori transparent, even from a perturbative viewpoint, in view of the non-trivial perturbative content of $\left.\hat{G}_{\varrho}\right)$ is shown:

$$
G=\lim _{\varepsilon \rightarrow 0}\left(\hat{G}_{\varepsilon}-\left[\hat{G}_{\varepsilon}\right]\right)+[F],
$$

where as before $\left[\hat{G}_{\varepsilon}\right]$ and $[F]$ are the values of $\hat{G}_{\varepsilon}$ and $F$ at $k=z=z^{\prime}=0$. Although $\hat{G}_{\varepsilon}$ and $\left[\hat{G}_{\varepsilon}\right]$ are not expected to be individually defined in the $\varepsilon \rightarrow 0$ limit, their difference is thus expected to have a well defined limit, equal to $G-[G]=G-[F]$. More precisely, our analysis introduces a family of approximations $G_{\varepsilon}$ of $G$, such that $F$ satisfies the expansion (4) with $\circ$ and $G$ replaced by $\circ_{\varepsilon}$ and $G_{\varepsilon}$, with

$$
G_{\varepsilon}-\left[G_{\varepsilon}\right]=\hat{G}_{\varepsilon}-\left[\hat{G}_{\varepsilon}\right], \forall \varepsilon
$$

and $\left[G_{\varepsilon}\right]=[F], \forall \varepsilon$, in view of the renormalization prescription.

Related versions of the analysis involving functions $F_{\varepsilon}$ depending on $\varepsilon$, will be mentioned in Sect. 3.2, where the converse derivation of the 2-particle irreducibility of $G$ from asymptotic completeness (through the previous results of [3] on $\hat{G}_{\varepsilon}$, Eq. (8) and assumptions on the $\varepsilon \rightarrow 0$ limit), and the justification of the heuristic procedure of [2] will also be outlined. Our alternative derivation of the renormalized equation is finally given in Sect. 3.3, as a consequence of Eqs. (2) and (8) (and up to assumptions on the $\varepsilon \rightarrow 0$ limit).

The analysis is presented for scalar theories, like $\phi_{4}^{4}$, that as is well known may not satisfy the axioms and whose existence itself is doubtful (see further analysis on this point in [11]). However, it can in fact be adapted to more realistic cases, e.g. renormalizable theories that exist and satisfy the axioms in space-time dimension 2 [11] such as the massive Gross-Neveu model, and it might possibly be adapted (with more work!) to more complicated physical theories in dimension 4.

\section{From Irreducibility to Asymptotic Completeness}

\subsection{Regularized Case}

As mentioned in Sect. 1, a quasi-equivalence is proved in [3] between the unitarity Eq. (3) and the 2-particle irreducibility of the kernels $\hat{G}_{\varrho}$ in the axiomatic sense. It 
follows there from an algebraic "intertwining formula," derived from (2), that relates the quantities $F_{+}-F_{-}-F_{+} * F_{-}$and $\left(\hat{G}_{\varrho}\right)_{+}-\left(\hat{G}_{\varrho}\right)_{-}$, whose vanishing means respectively Eq. (3) and the 2-particle irreducibility of $\hat{G}_{\varrho}$. The plus and minus signs refer to plus and minus $i \varepsilon$ boundary values in the region $(2 \mu)^{2}<s<(3 \mu)^{2}$. [The minus i\& quantities are obtained from the plus $i \varepsilon$ ones after one anticlockwise turn around $s=(2 \mu)^{2}$.] The proof is based on the relation (see [3]):

$$
\left(\circ_{\varrho}\right)_{+}-\left(\circ_{\varrho}\right)_{-}=*, \quad \forall \varrho \geqq 0,
$$

where $\left(\circ_{\varrho}\right)_{+}$and $\left(\circ_{\varrho}\right)_{-}$refer to integration contours $\Gamma_{+}(k)$ and $\Gamma_{-}(k)$.

We present below an alternative way of understanding how the irreducibility of $\hat{G}_{\varrho}$ yields Eq. (3), at least in the sense of formal expansions. To that purpose, we consider the Neumann series expansion of $F$ in Eq. (2):

$$
\begin{aligned}
F & =\sum_{n \geqq 0}\left(\hat{G}_{\varrho}\right)^{\circ}{ }^{(n+1)} \\
\equiv & \sum_{n \geqq 0} \underbrace{\hat{G}_{\varrho}{ }_{\varrho} \hat{G}_{\varrho} \cdots{ }_{\varrho} \hat{G}_{\varrho}}_{n+1 \text { factors } \hat{G}_{\varrho}} .
\end{aligned}
$$

The assumed analyticity of $\hat{G}_{\varrho}\left[\right.$ at $\left.s<(3 \mu)^{2}\right]$ and the relation (9) yield the discontinuity formula:

$$
\begin{aligned}
\Delta\left(\hat{G}_{\varrho^{\circ}}{ }^{(n+1)}\right) & \equiv\left(\hat{G}_{\varrho}^{\circ \varrho^{(n+1)}}\right)_{+}-\left(\hat{G}_{\varrho}^{\circ \varrho^{(n+1)}}\right)_{-} \\
& =\sum_{1 \leqq k \leqq n} \underbrace{\left(\hat{G}_{\varrho} \circ{ }_{\varrho} \hat{G}_{\varrho} \cdots \circ{ }_{\varrho} \hat{G}_{\varrho}\right)}_{k}+\underbrace{\left(\hat{G}_{\varrho} \circ{ }^{\circ} \cdots \circ_{\varrho} \hat{G}_{\varrho}\right)}_{n-k+1}-
\end{aligned}
$$

where $\left(\hat{G}_{\varrho} \circ_{\varrho} \ldots \circ_{\varrho} \hat{G}_{\varrho}\right)_{+} \equiv\left(\hat{G}_{\varrho}\right)_{+}\left(\circ_{\varrho}\right)_{+} \ldots\left(\circ_{\varrho}\right)_{+}\left(\hat{G}_{\varrho}\right)_{+}$, a similar relation holding with minus signs, and where $\left(\hat{G}_{\varrho}\right)_{+}=\left(\hat{G}_{\varrho}\right)_{-}$by assumption. Equation (11) follows from systematic cancellations, due to Eq. (9), between the terms in the last sum $\sum$.

Equation (11) yields in turn Eq. (3) in the sense of formal expansions in $\hat{G}_{\varrho}$ : namely the formal expansions of the two sides of Eq. (3) coincide as is easily checked. $\left(F_{+}=\sum_{n \geqq 0}\left(\hat{G}_{\varrho}^{\circ}{ }^{(n+1)}\right)_{+}, F_{-}=\sum_{n \geqq 0}\left(\hat{G}_{\varrho}^{\circ} \varrho^{(n+1)}\right)_{-}\right)$.

\subsection{Renormalized Case}

We first recall the definition of $\left(G \circ_{\varepsilon} \ldots \circ_{\varepsilon} G\right)_{r}$ for $\varepsilon \geqq 0$. It is for $\varepsilon>0$ a sum of terms associated with all possible sets $U$ (=forests in Zimmerman's terminology) of brackets that contain sequences of $\geqq 2$ factors $G$ and have no overlap. To each bracket [ ] is associated a constant, which is the value when all external energymomenta are fixed at zero $\left(k=z=z^{\prime}=0\right)$ of the function inside the brackets. The latter is the Feynman-type convolution product of factors which are either kernels $G$ or constants associated to subbrackets. For instance $\left[G \circ_{\varepsilon} G \circ_{\varepsilon} G\right]$ $=\left.\left(G \circ_{\varepsilon} G \circ_{\varepsilon} G\right)\right|_{k=z=z^{\prime}=0}$, where

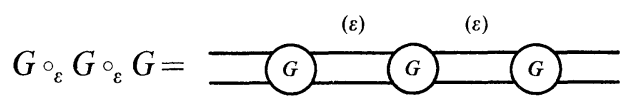


and $(\varepsilon)$ indicates regularization factors $\chi_{\varepsilon}$ for each internal line; $\left[G \circ_{\varepsilon} G \circ_{\varepsilon}\left[G \circ_{\varepsilon} G\right]\right]$ $=\left.\left(G \circ_{\varepsilon} G \circ_{\varepsilon}\left[G \circ_{\varepsilon} G\right]\right)\right|_{k=z=z^{\prime}=0}=\left[G \circ_{\varepsilon} G \circ_{\varepsilon} 1\right] \times\left[G \circ_{\varepsilon} G\right]$. Finally a multiplicative factor $(-1)^{N(U)}$, where $N(U)$ is the total number of brackets [ ] is included. E.g.

$$
\begin{gathered}
(G \circ G)_{r}=G \circ G-[G \circ G], \\
(G \circ G \circ G)_{r}=G \circ G \circ G-[G \circ G] \circ G-G \circ[G \circ G]-[G \circ G \circ G] \\
+[[G \circ G] \circ G]+[G \circ[G \circ G]],
\end{gathered}
$$

where $\varepsilon$ has been left implicit.

As in the case of renormalized Feynman integrals, the above definition is only symbolic at $\varepsilon=0$ : each individual term $G \circ G,[G \circ G], \ldots$ is expected to be infinite. $(G \circ \ldots \circ G)_{r}$ can be defined at $\varepsilon=0$, e.g. by first introducing, as in Zimmerman's approach, a renormalized integrand $[9,6]$, or alternatively by first considering the term $\left(G \circ_{\varepsilon} G \ldots \circ_{\varepsilon} G\right)_{r}$, and then letting $\varepsilon \rightarrow 0$. By an extension of the previous work [9], it is shown in [6] that, if $G$ is 2-particle irreducible (=analytic up to the 3-particle threshold), then $(G \circ \ldots \circ G)_{r}$ is well defined and analytic in a cut domain, (which contains euclidean space), with the cut along $s \geqq(2 \mu)^{2}$, and has also well defined boundary values $(G \circ \ldots \circ G)_{r}^{+}$and $(G \circ \ldots \circ G)_{r}^{-}$at $s>(2 \mu)^{2}$. This result relies on the assumption that the 2-point function $H_{2}$ (attached to each internal line in the convolution products) satisfies the axiomatic analyticity properties (with a pole in $s=k^{2}$ at $\mu^{2}$, where $\mu$ is the physical mass), and on "graduation assumptions" on both $H_{2}$ and $G$ : e.g. a decrease factor $\left(1+\left|z \pm z^{\prime}\right|\right)^{-1}$ is gained for each derivative of $G$ with respect to components of $z, z^{\prime}$, or of $k=p_{1}+p_{2}=p_{3}+p_{4}$. (See details in [6].)

We now wish to show that the irreducibility of $G$ also yields the unitarity Eq. (3), again in the sense of formal expansions, and to that purpose, we prove below the following discontinuity formula, which replaces (11):

$$
\Delta(\underbrace{G \circ G \ldots \circ G}_{n+1})_{r}=\sum_{1 \leqq k \leqq n}(\underbrace{G \circ \ldots \circ G}_{k})_{r}^{+} *(\underbrace{G \circ \ldots \circ G}_{n-k+1})_{r}^{-} .
$$

Equation (3) then follows, in the sense of formal expansions in $G$, in the same way as before, with (10) replaced by (4).

Proof of (14). We prove below (14) with $\circ$ replaced (everywhere) by ${ }_{\varepsilon}, \varepsilon>0$. Since $(G \circ \ldots \circ G)_{r}^{ \pm}=\lim _{\varepsilon \rightarrow 0}\left(G \circ{ }_{\varepsilon} \ldots{ }_{\varepsilon} G\right)_{r}^{ \pm}$by an adaptation of the methods of [6], Eq. (14) itself follows.

To that purpose, we shall use the formula (which is easily checked):

$$
(\underbrace{G \circ \circ_{\varepsilon} \ldots \circ_{\varepsilon} G}_{n+1})_{r}=\sum_{p \geqq 1} \sum_{\substack{n_{1}, \ldots, n_{p} \geqq 1 \\ \Sigma n_{l}=n+1}} \underbrace{\overline{[]_{\varepsilon}}}_{n_{1}} \circ_{\varepsilon} \underbrace{[]_{\varepsilon}}_{n_{2}} \ldots \circ_{\varepsilon} \underbrace{\overline{[]_{\varepsilon}}}_{n_{p}},
$$

where $\underbrace{[]_{\varepsilon}}_{n} \equiv G$ if $n=1$ and is, for $n>1$, the sum of all contributions to $(\underbrace{G \circ{ }_{\varepsilon} \ldots \circ{ }_{\varepsilon} G}_{n})_{r}$ in which a bracket $\left[\right.$ includes all factors $G$. E.g. it is equal to $-\left[G \circ_{\varepsilon} G\right]$ for $n=2$, and

$$
\underbrace{\overline{[]_{\varepsilon}}}_{n=3}=-\left[G \circ_{\varepsilon} G \circ_{\varepsilon} G\right]+\left[\left[G \circ_{\varepsilon} G\right] \circ_{\varepsilon} G\right]+\left[G \circ_{\varepsilon}\left[G \circ_{\varepsilon} G\right]\right] .
$$


A term in the sum of (15) is a Feynman type convolution integral of factors which are either kernels $G$ (if $n_{i}=1$ ) or constants $\overline{[]_{\varepsilon}}$ (if $n_{i}>1$ ). By using again Eq. (9), together with the assumed analyticity of $G$ (and the obvious analyticity of constants), its discontinuity is the sum of all terms obtained by replacing one of the operations ${ }_{\varepsilon}$ between two factors by $*$, with respective plus and minus $i \varepsilon$ boundary values on the left and right sides. The discontinuity of $\left(G \circ_{\varepsilon} \ldots{ }_{\varepsilon} G\right)_{r}$ is the sum of these discontinuities, i.e. is a sum of $*$ convolutions of left and right terms which are themselves plus and minus $i \varepsilon$ boundary values of Feynman-type convolutions of factors $G$ and $\overline{[]_{\varepsilon}}$. By regrouping all contributions with a given number $k$ of kernels $G$ on the left (and $n-k+1$ kernels $G$ on the right), including those inside the factors $\overline{[}_{\varepsilon}$, and by applying again Eq. (15) with $n+1$ replaced by $k$ or $n-k+1$, one reobtains exactly the term $(\underbrace{}_{\varepsilon} \circ_{\varepsilon} \ldots{ }_{\varepsilon} G)_{r}^{+} *(\underbrace{}_{\varepsilon} \ldots{ }_{\varepsilon} G)_{r}^{-}$. Equation (14) follows QED.

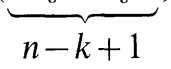

Note. After this work was completed, J. Bros has obtained an alternative proof of (14), which avoids our $\varepsilon \rightarrow 0$ limiting procedure, but uses more complicated partitions of contours.

The result of this subsection is an extension, in a simple case, of previous results of $[7,8]$, where unitarity is proved in fact [7] at each order in perturbation theory through a $T$-product formalism, a method which can also be adapted [8] to prove general discontinuity formulae on individual renormalized Feynman amplitudes. Our case corresponds to the graphs $>0<\ldots\rangle$ but with general irreducible kernels rather than constants at each vertex. Our procedure, in contrast to that of $[7,8]$ is first to establish the discontinuity formulae for individual terms, and then unitarity.

For a converse derivation of the irreducibility of $G$ from unitarity, see Remark 2 at the end of Sect. 3.2.

\section{Regularized and Renormalized Irreducible Kernels and Bethe-Salpeter Equations}

\subsection{Regularized Kernels}

The following link between two regularized kernels $\hat{G}_{\varrho}, \hat{G}_{\varrho^{\prime}}$ is proved by a direct adaptation of the proof of Lemma 4 in [10]:

$$
\hat{G}_{\varrho}-\hat{G}_{\varrho^{\prime}}=\hat{G}_{\varrho} x_{\varrho, \varrho^{\prime}} \hat{G}_{\varrho^{\prime}},
$$

where

$$
x_{\varrho, \varrho^{\prime}}=\circ_{\varrho}-\circ_{\varrho^{\prime}} .
$$

In view of Eq. (9), applied to ${ }^{\circ}$ and ${ }_{\varrho^{\prime}}$, the operator $x_{\varrho, \varrho^{\prime}}$ satisfies:

$$
\left(x_{\varrho, \varrho^{\prime}}\right)_{+}-\left(x_{\varrho, \varrho^{\prime}}\right)_{-}=0 \text {, }
$$

a relation which, together with (16), explains why various regularized kernels can be expected to be 2-particle irreducible (in the axiomatic sense) if one of them is.

\subsection{Regularized and Renormalized Kernels}

The formal Neumann series expansion of $\hat{G}_{\varrho}$ in Eq. (2) in terms of $F$ reads:

$$
\hat{G}_{\varrho}=\sum_{n \geqq 0}(-1)^{n} \underbrace{F \circ_{\varrho} F \ldots{ }_{\varrho} F}_{n+1 \text { factors } F},
$$


where $F$ can be expressed in terms of $G$ through Eq. (4). This gives the following formal expansion of $\hat{G}_{\varrho}$ in terms of $G$ :

$$
\begin{gathered}
\hat{G}_{\varrho}=\sum_{n \geqq 0}\left(\hat{G}_{\varrho}\right)_{n}, \\
\left(\hat{G}_{\varrho}\right)_{n}=\sum_{p \geqq 1} \sum_{\substack{n_{1}, \ldots, n_{p} \geqq 1 \\
\Sigma n_{i}=n+1}}(-1)^{p+1}(\underbrace{G \circ \ldots \circ G}_{n_{1}})_{r} \circ(\underbrace{G \circ \ldots \circ G}_{n_{2}})_{r} \cdots \circ \circ_{\varrho}(\underbrace{G \circ \ldots \circ G}_{n_{p}})_{r} .
\end{gathered}
$$

From a perturbative viewpoint, the content of $\hat{G}_{\varrho}$ follows from Eqs. (20), (21) by replacing $G$ by the sum of renormalized Feynman amplitudes of 2 p.i. graphs. It is not simple, even from a purely formal viewpoint at $\varrho=0$, and the irreducibility of $\left(\hat{G}_{\varrho}\right)_{n}$, i.e. of $\hat{G}_{\varrho}$ at each order, is not directly transparent. We explain it below, independently of the detailed perturbative background, starting from the assumption that $G$ is 2 particle irreducible (in the axiomatic sense). To that purpose, we first consider the functions $\left(\hat{G}_{\varrho}\right)_{n, \varepsilon}, \varepsilon>0$ defined by:

$$
\left(\hat{G}_{\varrho}\right)_{n, \varepsilon}=\sum_{p \geqq 1} \sum_{\substack{n_{1}, \ldots, n_{p} \\ \Sigma n_{i}=n+1}}(-1)^{p+1}(\underbrace{G \circ_{\varepsilon} \ldots \circ_{\varepsilon} G}_{n_{1}})_{r} \circ_{\varrho} \cdots \circ_{\varrho}(\underbrace{G \circ \ldots \circ G}_{n_{p}})_{r},
$$

i.e. the operator $\circ$ has been replaced by ${ }_{\varepsilon}$ everywhere in the right-hand side of $(21)$. From Eq. (15), the following expression of $\left(\hat{G}_{\varrho}\right)_{n, \varepsilon}$ is easily checked:

$$
\left(\hat{G}_{\varrho}\right)_{n, \varepsilon}=\sum_{p \geqq 1} \sum_{\substack{n_{1}, \ldots, n_{p} \geqq 1 \\ \Sigma n_{i}=n+1}} \underbrace{\overline{[]_{1}}}_{n_{1}} x_{\varepsilon, \varrho} \ldots x_{\varepsilon, \varrho} \underbrace{\overline{[]_{1}}}_{n_{p}} \varepsilon,
$$

where, in accordance with (17), (18), $x_{\varepsilon, \varrho}={ }_{\varepsilon}-{ }_{\varrho}{ }_{\varrho}$ satisfies Eq. (18). The 2-particle irreducibility of $\left(\hat{G}_{\varrho}\right)_{n, \varepsilon}, \forall \varepsilon>0$ can then be proved on the basis of this result and of the fact that all factors []$_{\varepsilon}$ in (23), which are either equal to $G$ or constants, are analytic (i.e. satisfy more precisely the axiomatic 2-particle irreducibility).

Coming back to the expression (22), it should be shown by methods of [6], as we shall admit here, that the functions $\left(\hat{G}_{\varrho}\right)_{n, \varepsilon}$ are uniformly bounded in adequate regions and converge to $\left(\hat{G}_{\varrho}\right)_{n}$ in the $\varepsilon \rightarrow 0$ limit, this limit being then itself 2-particle irreducible. Q.E.D.

The kernel $\hat{G}_{\varrho}$ is not defined at $\varrho=0$. However, a purely algebraic calculation at $\varrho=0$ gives:

$$
\hat{G}_{0}=G+\overline{[G \circ G]}+\overline{[G \circ G \circ G]}+\ldots
$$

This is in fact the particular case $\varrho=\varepsilon=0$ of formula (23), with $x_{\varepsilon, \varrho}=x_{\varepsilon}-x_{\varrho}=0$ for $\varrho=\varepsilon$. From Eq. (24), one finds in turn:

$$
\hat{G}_{0}-\left[\hat{G}_{0}\right]=G-[G] \text {, }
$$

which suggests that, although $\hat{G}_{0}$ and $\left[\hat{G}_{0}\right]($ like $\overline{[G \circ G]}, \ldots)$ are expected to be infinite, their difference is a meaningful finite quantity, equal to $G-[G]$. This result is confirmed in the following more precise way:

$$
\lim _{\varepsilon \rightarrow 0}\left(\hat{G}_{\varepsilon}-\left[\hat{G}_{\varepsilon}\right]\right)=G-[G],
$$

by writing $F$ (see below) in the (formal) form analogous to (4):

$$
F=\sum_{n \geqq 0}\left(G_{\varepsilon} \circ_{\varepsilon} G_{\varepsilon} \ldots \circ_{\varepsilon} G_{\varepsilon}\right)_{r}
$$


if $\lim _{\varepsilon \rightarrow 0} G_{\varepsilon}=G$. In fact, the same algebraic analysis as above (with now no infinities at $\varepsilon>0$ ) gives:

$$
\begin{gathered}
\hat{G}_{\varepsilon}=G_{\varepsilon}+\sum_{n \geqq 2} \underbrace{\overline{\left[G_{\varepsilon} \circ G_{\varepsilon} \circ{ }_{\varepsilon} \ldots \circ_{\varepsilon} G_{\varepsilon}\right]}}_{n \text { factors } G_{\varepsilon}}, \\
\hat{G}_{\varepsilon}-\left[\hat{G}_{\varepsilon}\right]=G_{\varepsilon}-\left[G_{\varepsilon}\right],
\end{gathered}
$$

from which Eq. (26) follows.

Equation (8) of Sect. 1 is thus obtained, since $\left[G_{\varepsilon}\right]=[F]$ in view of the renormalization prescription. $\left[\left(G_{\varepsilon} \circ_{\varepsilon} \cdots{ }_{\varepsilon} G_{\varepsilon}\right)_{r}\right.$ is equal to zero at $k=z=z^{\prime}=0$. ] Finally, one checks (see [12]) that $F$ does admit the expansion (27) with:

$$
G_{\varepsilon}=\hat{G}_{\varepsilon}-\left[\hat{G}_{\varepsilon}\right]+[F] .
$$

We note that the "inverse" of Eq. (28), e.g. derived from (29) and from (10) (considered at zero external momenta) is:

$$
G_{\varepsilon}=\hat{G}_{\varepsilon}+\sum_{n \geqq 2}[\underbrace{\hat{G}_{\varepsilon} \circ_{\varepsilon} \hat{G}_{\varepsilon} \ldots \circ_{\varepsilon} \hat{G}_{\varepsilon}}_{n}] .
$$

We now make the following comments:

1) Various related versions of the above analysis can be given, e.g. by introducing functions $F_{\varepsilon}$ which, from the perturbative viewpoint, may be sums of all renormalized Feynman amplitudes in which cut off factors $\chi_{\varepsilon}(k)$ are attached to each internal line $\left(\lim _{\varepsilon \rightarrow 0} F_{\varepsilon}=F\right) \cdot G_{\varepsilon}$ is then similarly the sum of renormalized Feynman amplitudes of 2 p.i. Feynman graphs with the same regularization factors. The algebraic analysis is identical to that above with $F$ replaced by $F_{\varepsilon}$.

2) The converse of Sect. 2.2 (from unitarity to the irreducibility of $G$ ) can be obtained from (30), which yields the irreducibility of $G_{\varepsilon}$ from that already established [3] for the regularized kernels $\hat{G}_{\varepsilon}$, from the assumption that $\lim G_{\varepsilon}=G$ and from further assumptions of uniform bounds on $G_{\varepsilon}$ (i.e. on $\hat{G}_{\varepsilon}-\left[\hat{G}_{\varepsilon}\right]$ ) when $\varepsilon \rightarrow 0$. The latter can be viewed as associated with the class of theories considered.

3) The following heuristic derivation of integral equations between $F$ and differences of values of $G$ is given in [2]. Starting from the usual Bethe-Salpeter Eq. (1) which, as already mentioned, cannot be expected to be valid by itself (even with possibly some regularization), these new equations are derived formally. Individual terms in the new equations can now be expected to be well defined in view of the assumptions on the decrease of differences of values of $G$, and these equations can be verified in perturbation theory (although the verification is not given explicitly in [2]). Thus, they can be assumed to hold.

A more explicit and satisfactory justification of this procedure follows from our analysis: one may start for $\varepsilon>0$ from a regularized form of $(1)$ involving a kernel $\hat{G}_{\varepsilon}$ different from $G$ (or $G_{\varepsilon}$ ). However, in any derived equation, differences of values of $\hat{G}_{\varepsilon}$ at various points can be replaced in view of (29) by differences of values of $G_{\varepsilon}$, and hence of $G$ in the $\varepsilon \rightarrow 0$ limit.

\subsection{Derivation of the Renormalized Bethe-Salpeter Equation}

We first consider $\varepsilon>0$ and show below from Eqs. (2) (at $\varrho=\varepsilon$ ) and (30) that

$$
F=G_{\varepsilon}+F \circ_{\varepsilon} G_{\varepsilon}-\Lambda_{\varepsilon}\left[F \circ_{\varepsilon} G_{\varepsilon}\right] \text {. }
$$


The renormalized Eq. (5) follows if $G_{\varepsilon}$ is assumed to have a limit $G$ when $\varepsilon \rightarrow 0$ and up to details not to be discussed here.

Proof of (32). Equation (2) and the relation:

$$
\hat{G}_{\varepsilon}=G_{\varepsilon}-\left([F]-\left[\hat{G}_{\varepsilon}\right]\right)
$$

derived from (30) yield:

$$
F=G_{\varepsilon}+F \circ_{\varepsilon} G_{\varepsilon}-\left([F]-\left[\hat{G}_{\varepsilon}\right]\right)\left(1+F \circ_{\varepsilon} 1\right) .
$$

On the other hand, Eq. (2) restricted at $k=z=z^{\prime}=0$ gives:

$$
[F]-\left[\hat{G}_{\varepsilon}\right]=\left[F \circ_{\varepsilon} \hat{G}_{\varepsilon}\right] \text {. }
$$

The relation

$$
F \circ_{\varepsilon} \hat{G}_{\varepsilon}=F \circ_{\varepsilon} G_{\varepsilon}-\left[F \circ_{\varepsilon} \hat{G}_{\varepsilon}\right]\left(F \circ{ }_{\varepsilon} 1\right)
$$

derived from (33), (35) then gives, at $k=z=z^{\prime}=0$ :

$$
\left[F \circ_{\varepsilon} \hat{G}_{\varepsilon}\right]\left(1+\left[F \circ_{\varepsilon} 1\right]\right)=\left[F \circ_{\varepsilon} G_{\varepsilon}\right] \text {. }
$$

Equation (32) follows. Q.E.D.

As in Sect. 3.2, the same algebraic derivation holds with $F$ replaced by $F_{\varepsilon}$. Acknowledgements. I am pleased to thank Prof. J. Bros for kindly communicating to me his first results on the renormalized Bethe-Salpeter formalism prior to publication and for his explanations on the previous work by Symanzik.

\section{References}

1. Symanzik, K.: J. Math. Phys. 1, 249 (1960)

2. Symanzik, K.: Infrared singularities and small-distance-behaviour analysis. Commun. Math. Phys. 34, 7 (1973)

3. Bros, J.: Analytic methods in mathematical physics, p. 85. Gilbert, R., Newton, R. (eds.). New York: Gordon and Breach 1970

Bros, J., Lassalle, M.: Analyticity properties and many-particle structure in general quantum field theory. III. Two-particle irreducibility in a single channel. Commun. Math. Phys. 54, 33 (1977)

4. Bros, J.: $r$-Particle irreducible kernels, asympotic completeness and analyticity properties of several particle collision amplitudes. Physica 124 A, 145 (1984), and in preparation

5. Iagolnitzer, D.: Unitarity or asymptotic completeness equations and analytic structure of the $S$ matrix and green functions. In: Proceedings of the 1983 Brasov Summer Institute (to be published) and Saclay preprint SPhT/85-034

6. Bros, J., Ducomet, B.: In preparation

7. Epstein, H., Glaser, V.: Statistical mechanics and quantum field theory, p. 501. Stora, R. (ed.). New York: Gordon and Breach 1971

8. Epstein, H., Glaser, V.: Private communication of H. Epstein

9. Bros, J., Manolessou-Grammaticou, M.: Renormalized $G$-convolution of $N$-point functions in quantum field theory: Convergence in the Euclidean case I and II. Commun. Math. Phys. 72, 175 and 207 (1980)

10. Iagolnitzer, D.: Irreducible kernels and nonperturbative expansions in a theory with pure $m \rightarrow m$ interaction. Commun. Math. Phys. 88, 235 (1983)

11. Magnen, J., Rivasseau, V., Sénéor, R.: Private communication

12. Iagolnitzer, D.: Appendix to SPhT report 84-99 (unpublished)

Communicated by K. Osterwalder

Received August 20, 1984; in revised form January 22, 1985 